\title{
ANÁLISE DA GOVERNANÇA PORTUÁRIA A PARTIR DA PATH DEPENDENCE THEORY: UM ESTUDO JUNTO AO PORTO DE VALÊNCIA
}

\section{PORT GOVERNANCE ANALYSIS FROM PATH DEPENDENCE THEORY: A STUDY ON THE VALENCIA PORT}

\author{
Guilherme Bergmann Borges Vieira ${ }^{1}$; Roberto Birch Gonçalves ${ }^{2}$; Gabriel Sperandio Milan³ \\ Francisco José Kliemann Neto ${ }^{4}$ \\ ${ }^{1}$ Universidade de Caxias do Sul - UCS - Caxias do Sul - RS - Brasil \\ gbbvieir@ucs.br \\ ${ }^{2}$ Universidade de Caxias do Sul - UCS - Caxias do Sul - RS - Brasil \\ rbgoncal@ucs.br \\ ${ }^{3}$ Universidade de Caxias do Sul - UCS - Caxias do Sul - RS - Brasil \\ gabmilan@terra.com.br \\ ${ }^{4}$ Universidade Federal do Rio Grande do Sul - UFRS - RS - Brasil \\ kliemann@producao.ufrgs.br
}

\begin{abstract}
Resumo
Os portos vêm sendo cada vez mais estudados como aglomerados empresariais e um aspecto emergente na literatura é a coordenação dos atores pertencentes a estes aglomerados, ou seja, a governança portuária. Parte dos estudos busca relacionar o modelo de governança portuária com a performance dos portos, mas os resultados não são conclusivos, uma vez que a performance portuária também depende de uma série de outros fatores. Entre tais fatores estão os eventos passados, as escolhas anteriores feitas pelo porto, que impactam na sua competitividade presente $e$ futura. Portanto, a governança portuária pode ser estudada a partir da perspectiva da dependência da trajetória (Path Dependence). Assim, o presente estudo teve como objetivo analisar as inovações relacionadas à governança portuária no Porto de Valência (Espanha) a partir dos pressupostos da Path Dependence Theory. Os resultados indicam que o Porto vem obtendo resultados positivos como decorrência de suas ações de governança. No entanto, sugere-se um constante monitoramento ambiental para evitar um possível 'trancamento' do Porto em uma trajetória desfavorável.
\end{abstract}

Palavras-chave: governança portuária; path dependence theory; porto de valência.

\section{Introdução}

Devido a sua importância para o comércio exterior, atuando como elos de conexão terramar, e para o desenvolvimento das regiões em que estão inseridos, os portos vem sendo cada vez mais estudados, especialmente nas últimas duas décadas. Parte destes estudos contempla uma perspectiva mais ampla, a qual considera o porto como uma aglomeração empresarial. Nesse contexto, Geiger (2009) afirma que o estudo de aglomerações empresariais pode ser classificado a 
partir de duas lógicas principais: a do sequenciamento das atividades ou lógica funcional; e a do desenvolvimento do território ou lógica territorial. No primeiro caso, enquadram-se os estudos de 'cadeias produtivas' e de 'cadeias de valor', enquanto no segundo, classificam-se os estudos de 'arranjos produtivos' e de clusters.

Nos estudos portuários, por sua vez, é comum a utilização dos termos 'cluster portuário' (DE LANGEN, 2004), 'comunidade portuária' (MARTIN; THOMAS, 2001) e, em menor grau, 'cadeia logístico-portuária' (LÓPEZ; POOLE, 1998). No entanto, não é facilmente percebida a diferenciação entre a lógica funcional e a territorial nesses estudos, encontrando-se uma série de trabalhos que mencionam o termo cluster, mas consideram como foco de análise o sequenciamento de atividades.

De qualquer modo, independentemente da abordagem, os portos vem sendo cada vez mais estudados como uma aglomeração de atores, sendo que um aspecto emergente diz respeito à coordenação destes atores, ou seja, a governança portuária. O número de estudos dedicado ao tema é relativamente escasso e fortemente concentrado a partir da segunda metade da década relativa aos anos 2000. Parte destes estudos busca relacionar a governança, e especialmente o modelo de governança portuária, com a performance dos portos (BALTAZAR; BROOKS, 2006; BROOKS; CULLINANE, 2006).

Os resultados, no entanto, permanecem inconclusivos, o que permite afirmar que a governança portuária é importante, mas a performance do porto depende também de inúmeros outros fatores. Entre os fatores que podem influenciar o desempenho portuário estão os eventos passados, as escolhas anteriores feitas pelo porto e que impactam sua competitividade presente e futura. Portanto, a governança portuária pode ser estudada a partir da perspectiva da dependência da trajetória ou Path Dependence.

Dado esse contexto, o presente estudo foi norteado pela seguinte questão central de pesquisa: Quais são os principais eventos associados à governança portuária no contexto do Porto de Valência (Espanha) e suas possíveis consequências para a cadeia logístico-portuária do referido porto pela ótica da Teoria da Path Dependence? Para responder a tal questão, foi estabelecido como objetivo do estudo analisar as inovações relacionadas à governança portuária em Valência a partir dos pressupostos da Path Dependence. A escolha pelo Porto de Valência, como unidade de análise, justifica-se pelo fato de o mesmo ter sido considerado Best-in-Class em clusters portuários pelo Port Cluster Governance Committee (PCGC) do Global Institute of Logistics (GLI).

Além disso, cabe ressaltar que o trabalho está estruturado em quatro seções. A primeira seção apresenta uma discussão sobre o Referencial Teórico a respeito dos fundamentos da Teoria da Path Dependence e do conceito de governança portuária, apresentando os principais modelos de governança encontrados na literatura. A segunda seção, por sua vez, apresenta os procedimentos 
metodológicos utilizados no estudo. A terceira seção traz a análise da governança portuária exercida no Porto de Valência pela perspectiva da Teoria da Path Dependence. E, por fim, na quarta seção, são apresentadas algumas considerações finais e sugestões de estudos futuros.

\section{Referencial teórico}

Neste capítulo são apresentados os principais conceitos associados à Path Dependence e à governança portuária. Busca-se identificar a potencial contribuição da abordagem da Path Dependence para o entendimento da governança portuária, especialmente no que diz respeito às potenciais consequências da dependência da trajetória no modelo de governança portuária e, consequentemente, na performance do porto.

\subsection{A teoria da Path Dependence}

Inicialmente, cabe ressaltar que, neste estudo, os termos 'dependência de caminho' e 'dependência da trajetória' são utilizados como sinônimos para representar a Teoria da Path Dependence (NELSON; WINTER, 1982; ARTHUR, 1994; DOSI; NELSON, 1994; DAVID, 2000). A lógica por trás da Path Dependence é a consideração de que a história importa, que decisões anteriores poderão ter consequências aos fatos presentes, por vezes 'trancando' ou 'aprisionando' (lock-in, no termo inglês) a empresa (neste caso, o Porto) em um curso irreversível. Segundo Licha (2004, p.107), “essa irreversibilidade é a chave para o conceito de dependência da trajetória, já que uma vez que o processo econômico entrou numa certa rota existem forças internas que fazem com que não seja possível abandoná-la espontaneamente".

A Teoria Path Dependence, conforme Liebowitz e Margolis (1995), portanto, mostra uma perspectiva alternativa para a análise de unidades econômicas, como uma reformulação revolucionária do paradigma neoclássico, que geralmente desconsidera todo um conjunto de decisões e de aprendizagem acumulado pelas organizações.

Consoante isso, Veblen (1990) estudou as razões que levaram a indústria alemã a superar a indústria britânica. A razão principal foi devido à indústria britânica ter se desenvolvido primeiro e ao seu redor terem sido criadas uma série de complementaridades entre os atores. Este fato 'prendeu' (lock-in) a indústria britânica em uma tecnologia obsoleta, enquanto a Alemanha pôde investir em novas e mais eficientes tecnologias, tendo um resultado superior. Neste sentido, a ideia principal da teoria evolucionista se encaixa, pois busca explicar algo na sua forma dinâmica, ou seja, os acontecimentos ao longo do tempo. Busca entender os 'porquês' de algo ter acontecido, porque aconteceu e qual foi o percurso realizado até aquele momento (DOSI; NELSON, 1994). Para a teoria evolucionista, adquire-se determinada competência como resultado de um processo histórico de escolhas. Em síntese, se mudanças dentro do campo da economia ocorrem o tempo 
todo, é de se supor que algum tipo de processo evolucionário esteja ocorrendo, tal qual a visão Darwiniana da evolução da vida (ou das espécies). Justamente sobre esta visão de acontecimentos históricos, David (2000) faz um alerta aos economistas: a de que alguns deles têm incapacidade de aprender e ver como os acontecimentos históricos podem causar influência sobre os resultados posteriores, podendo influenciar consideravelmente os paradigmas econômicos.

O conceito base da Path Dependence foi elaborado por Arthur (1994). O autor analisou o processo de tomada de decisão e os motivos que levavam as empresas a localizarem novas unidades em determinados locais. Na concepção deste autor, a forma clássica de se entender esse processo, baseada no retorno apenas, não explicava todas as decisões e implicações. Era preciso, portanto, ampliar a perspectiva. $\mathrm{O}$ autor utilizou a análise de escolhas e ações anteriores para justificar ações posteriores, nas quais fica claro que a história ocorrida importa, influenciando eventos posteriores.

Nesta mesma linha de raciocínio, Licha (2004, p. 107) reforça, dizendo que "o método proposto por Arthur (1994) para estudar a dinâmica do sistema é analisar as suas tendências de longo prazo", ou seja, a análise se procede após o transcurso de um número suficientemente elevado de períodos, de forma a eliminar o efeito das flutuações de curto prazo. Este fato adiciona definitivamente os aspectos temporais e seus efeitos para que se possa fazer uma análise adequada. Ainda para Licha (2004, p.107), "se a evolução de um processo está condicionada pela sua própria história então ele é dependente da trajetória"(...) "neste caso, um mesmo processo pode dar lugar a múltiplas estruturas no longo prazo em função dos eventos históricos acontecidos".

De fato, para Robinson e Meier (2006), os modelos de dependência de caminho vão além, explicando que as condições históricas influenciam a tomada de decisão e que a história gera efeitos na decisão contemporânea, tudo isso contextualizado dentro do dinamismo que a teoria evolucionária apregoa. Segundo Liebowitz e Margolis (1995), o pressuposto da dependência de caminho é que uma pequena ou passageira vantagem, ou aparentemente inconsequente modificação, conduzida por alguma tecnologia, produto ou padrão, pode ter importante ou irreversível influência na alocação de recursos futuros, mesmo em um mundo caracterizado por decisões voluntárias, com um comportamento individual maximizador.

Inclusive, Wilk (1997) destaca que a dependência de caminho acontece quando uma habilidade ou competência foi desenvolvida e acumulada ao longo do tempo, em uma sequência de aprendizado, envolvendo um processo de acertos e erros que não pode ser imitado rapidamente pelos competidores. O autor afirma, ainda, que os recursos estratégicos ou mesmo as competências essenciais são produto de acertos e erros desenvolvidos e acumulados no decorrer do tempo.

Em acréscimo, conforme Liebowitz e Margolis (1995), o efeito de decisões e das mudanças ocorridas no passado (conduzidas por tecnologia, produtos e/ou serviços ou algum tipo de padrão) pode influenciar de forma irreversível e intensa os recursos. Diante disso, pode-se concluir que os 
eventos intertemporais afetam sobremaneira os recursos, em especial os recursos principais geradores de uma vantagem competitiva sustentável. Arthur $(1989 ; 1994)$ e Liebowitz e Margolis (1995) admitem quatro causas de processos Path Dependence que, embora relacionadas, são diferentes: i) aumento de retornos; ii) autor-reforço; iii) feedback positivo; e iv) 'trancamento' ou 'aprisionamento' (lock-in).

É imperioso ressaltar um cuidado que as empresa devem ter, pois, de acordo com Mueller (1997), empresas que possuem o fenômeno interno de 'cultivar-deliberadamente' a Path Dependence não produzem algum tipo de vantagem competitiva sustentável. O autor explica que os primeiros a se moverem acabam tendo desvantagem no futuro por cultivarem em excesso as coisas que deram certo no passado sem capitalizar seus benefícios. Salienta, ainda, que a listas dos que deram errado por cultivar o passado é bem maior dos que deram certo. Ainda para Mueller (1997), uma dependência de trajetória originada externamente é melhor do que uma interna.

Para verificar e analisar a relação de acontecimentos sob o ponto de vista da Path Dependence, pode-se propor uma abordagem sob duas dimensões: i) a verificação da tipologia da Path Dependence (LIEBOWITZ; MARGOLIS, 1995), na qual pela identificação do tipo pode-se tomar decisões que previnam efeitos negativos de decisões do passado; e ii) pelos efeitos (ARTHUR 1989; 1990; 1994, LIEBOWITZ; MARGOLIS, 1995), pelos quais se pode analisar a continuidade de algumas decisões, tendo-se clareza de que complementaridades acontecem e quem mais é afetado pelo processo.

Neste horizonte, Liebowitz e Margolis (1995) propuseram uma classificação em três graus da Path Dependence, relacionando a identificação do tipo com as possíveis decisões de tal forma que estas previnam os efeitos negativos do lock-in promovidos pelas decisões passadas: Primeiro Grau: é a simples assertiva de que existe relação intertemporal, mas a dependência das condições iniciais não resulta em qualquer ineficiência. Neste grau, praticamente não há implicações maiores a serem estudadas; Segundo Grau: uma ação tomada hoje tem acontecimentos posteriores que se revelam inferiores ao que foi pensado, devido, principalmente, à assimetria de informações no momento de tomar a decisão, mas custo de reverter a ação é muito alto; e Terceiro Grau: é similar ao anterior, mas neste caso é possível reverter uma escolha cujo resultado se mostre ineficiente, a partir de correções e melhorias na trajetória.

Ao se analisar os efeitos da Path Dependence propostos por diversos autores (ARTHUR 1989; 1190; 1994; 1996, LIEBOWITZ; MARGOLIS, 1995), pode-se concluir sobre a continuidade de algumas decisões e se ter clareza de quais complementaridades acontecem e quais os seus efeitos. Os efeitos podem ser vistos em quatro aspectos, a saber: i) Aumento dos retornos: significa que quanto mais uma escolha é feita ou ação é tomada, mais aumenta seus benefícios. Emergem de um processo virtuoso no qual a expansão do uso de certo fator faz com que se torne cada vez mais 
vantajoso usá-lo (LICHA, 2004); ii) Auto-reforço: significa que fazendo uma escolha ou uma ação, coloca-se numa posição em que um conjunto de forças ou instituições complementares encorajam aquela escolha a se sustentar. $\mathrm{O}$ desenvolvimento de parceiros e complementaridades com foco nas ações já definidas se ampliam de forma contínua; iii) Feedback positivo: significa que fazendo uma escolha ou ação, criam-se externalidades positivas (efeitos do beneficio sobre terceiros sem que os mesmos incorram em custos associados a essa ação). A análise desse aspecto permite compreender como as externalidades surgem e são disseminadas, fazendo com que se possa interferir nesse processo e se abastecer dessas informações; e iv) Lock-in: significa que uma escolha ou ação tornase melhor do que qualquer outra, uma vez que um número suficiente de pessoas já fez essa escolha. Quando uma estrutura é alcançada o processo não pode sair dela de forma endógena (sem intervenção externa), então existe certa rigidez estrutural e o processo está bloqueado (LICHA, 2004).

\subsection{Governança portuária}

Em termos de extensão e pelo tipo de atividades que realiza, a indústria portuária teve sempre significativas repercussões econômicas e sociais nas regiões em que está estabelecida, contribuindo com o seu desenvolvimento e com a geração de riquezas (VIEIRA et al, 2010; MILAN; VIEIRA, 2011). Os portos, embora tenham evoluído nos seus vários estágios de simples pontos de carga e descarga dos bens a complexos industriais com infra-estrutura física compatível a um hub (ponto de concentração) da cadeia de transporte, continuam a evoluir conforme o ambiente de operação (VIEIRA et al, 2010; MILAN; VIEIRA, 2011).

Atualmente, os portos são organizados em plataformas logísticas, agindo como interfaces

entre o sistema de produção e os centros de consumo, atuando com a finalidade de eliminar a descontinuidade entre o modal marítimo e o transporte terrestre. Ao mesmo tempo, os portos estão se transformando em um componente estratégico para a atuação dos principais protagonistas da comunidade portuária, que inclui, entre outros, as autoridades portuárias, os operadores de terminais e os agentes de carga (VIEIRA et al, 2010; MILAN; VIEIRA, 2011). Isto porque o porto passou a ser um elemento-chave na criação de redes para desenvolver e realizar as atividades de tais agentes (SUYKENS; van de VOORDE, 1998; MEERSMAN; MOGLIA; van de VOORDE, 1999; HEAVER et al, 2000; NOTTEBOOM; WINKELMANS, 2001; van de VOORDE; WINKELMANS, 2002; WINKELMANS, 2008).

O processo de planejamento portuário desempenha um papel-chave na determinação da posição de um porto na hierarquia marítima (FRANKEL, 1989; UNCTAD, 1993). Uma das mudanças importantes que se tem identificado nos anos recentes é a expansão do porto além de seus limites históricos. Ou seja, o ambiente portuário atual não se restringe mais à concepção clássica, 
estendendo-se além dos limites locais (AKABANE; GONÇALVES; SILVA, 2008). Para Akabane, Gonçalves e Silva (2008), a autoridade portuária do passado não pode mais dar respostas adequadas ao cenário atual em que estão inseridos os portos, caracterizado por redes de negócios de alcance global e complexos sistemas logísticos, desenvolvidos em uma conjuntura de elevada incerteza (NOTTEBOOM, 2007).

Para responder efetivamente às atuais dinâmicas do mercado, é necessário o exame de uma situação nova, em que a autoridade portuária terá a necessidade de negociar com outras entidades e instituições, estando mais vulnerável em função de uma maior interferência externa e uma menor independência (AKABANE; GONÇALVES; SILVA, 2008). Em resumo, o foco da competitividade portuária vem gradualmente se deslocando do porto em si para o conjunto da comunidade, cluster ou cadeia portuária, ou seja, para o aglomerado empresarial em que o porto está inserido.

Na perspectiva de Winkelmans (2008), os diferentes atores do cluster portuário são todos os indivíduos ou grupos que têm interesse ou são afetados pelas atividades portuárias. Tais indivíduos ou grupos podem ser classificados em quatro categorias: i) os stakeholders internos, que são os gestores do porto, os colaboradores do porto, os membros do conselho de administração e os shareholders; ii) os players (atores) do mercado, que contemplam os órgãos de gestão de mão-deobra avulsa, os operadores de transporte ferroviário, rodoviário e de cabotagem, as companhias de navegação e seus agentes, os freight forwarders (agentes internacionais de carga) e demais prestadores de serviços logísticos, os práticos, os rebocadores, os embarcadores e consignatários de cargas etc.; iii) a administração pública, que inclui os diferentes departamentos de governo em nível local, regional, nacional e supranacional; e iv) a comunidade em geral (moradores do entorno portuário, consumidores, grupos de pressão ambiental etc.). E é neste contexto que está inserida a governança portuária.

A governança conta com uma ampla diversidade de conceitos, podendo ser entendida como (GEIGER, 2009): i) um padrão de comportamento do estado ou das empresas; ii) o regramento ou a coordenação das transações entre as empresas; ou iii) a direção e coordenação de atores pertencentes a aglomerados empresariais. O presente estudo está associado à terceira definição, podendo-se entender a governança como um sistema de regras formais e informais que estabelecem as pautas de interação entre atores no processo de tomada de decisão.

Quanto ao conceito de governança corporativa, a Organização para a Cooperação e Desenvolvimento Econômico (OCDE, 2004) a define como um sistema por meio do qual as corporações são direcionadas e controladas. A estrutura de governança corporativa especifica a distribuição de direitos e de responsabilidades entre os diferentes participantes da 'corporação' e dita as normas e procedimentos para o processo de tomada de decisão em relação aos assuntos corporativos. Dessa forma, é provida a estrutura por meio da qual os objetivos da corporação são 
estabelecidos, os meios para alcançar tais objetivos são definidos e as formas de aferição de performance são delineadas. A partir dos conceitos anteriores, é possível distinguir dois tipos de governança portuária: a governança da Autoridade Portuária (governança corporativa) e a governança do aglomerado portuário (governança da comunidade, cluster ou cadeia portuária). $\mathrm{O}$ presente estudo relaciona-se com esse segundo tipo de governança portuária (governança do aglomerado portuário).

De acordo com Brooks (2004), a governança deve ser considerada uma das prioridades da gestão portuária nos próximos anos. Essa idéia também é corroborada pela Conferência Européia de Portos Marítimos - The European Seaports Conference (ESPO, 2008), que decidiu estabelecer um comitê para tratar especificamente do assunto, entendendo que os portos precisam ser coordenadores dinâmicos da comunidade portuária, agindo como facilitadores da cadeia logística. E isso deve ser pensado não só em relação ao microambiente (clientes, operadores de terminais, agentes logísticos e outros provedores de serviço), mas também no que se refere ao macroambiente e às relações governos (locais e regionais), sociedade e demais stakeholders envolvidos.

O principal argumento para um envolvimento mais ativo da autoridade portuária na coordenação dos aglomerados portuários é o fato de que a eficiência na cadeia logística não emerge espontaneamente. Portanto, uma maior coordenação (governança) dos atores pertencentes a essa aglomeração, fomentada pela autoridade portuária, pode tornar os portos e as cadeias logísticas mais eficientes (DE LANGEN; NIJDAM; VAN DER HORST, 2007). Mas, para que isso aconteça, é necessário pensar em ações práticas que impulsionem a coordenação ou governança como suporte à referida eficiência portuária.

Tanto a implementação de ações de governança quanto seus resultados são condicionados por decisões anteriores que podem gerar consequências presentes e futuras, 'trancando' (lock-in) o porto em determinado curso, que pode ser favorável ou não ao desempenho portuário. É neste ponto a governança portuária e a teoria da dependência da trajetória (Path Dependence) se encontram.

O principal modelo de governança portuária encontrado na literatura é o de Baltazar e Brooks (2006), denominado Matching Framework. O modelo está baseado, em essência, em três elementos: i) ambiente em que está inserido o porto; ii) a estratégia estabelecida; e iii) a estrutura. Estes três elementos, em seu conjunto, definem o desempenho portuário (Figura 1). 


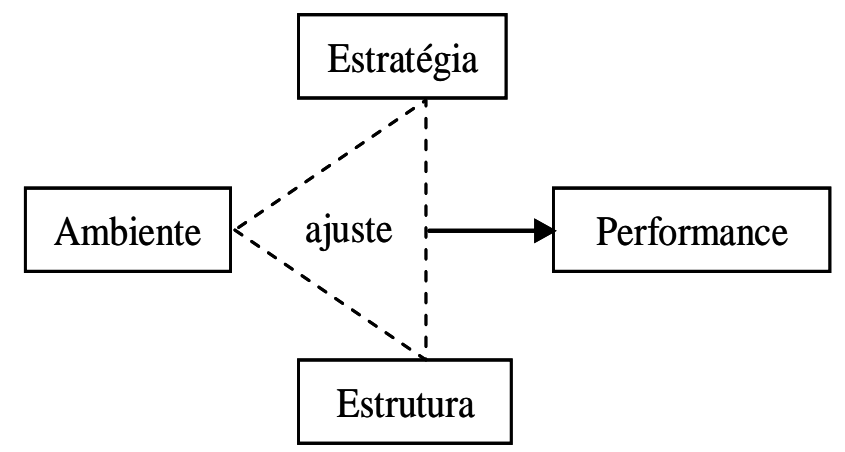

Fonte: Adaptado de Baltazar e Brooks (2006)

Segundo Baltazar e Brooks (2006), o ambiente em que está inserido o porto pode ter maior ou menor grau de incerteza, em função da complexidade e do dinamismo existentes; já a estratégia competitiva pode ser defensiva ou prospectiva (MILES; SNOW, 1978 apud BALTAZAR; BROOKS, 2006); e a estrutura pode ser mais ou menos centralizada e os processos desenvolvidos podem ter maior ou menor padronização. Baltazar e Brooks (2006) propõem no modelo a idéia de ajuste (fit) entre ambiente, estratégia e estrutura, com vistas a gerar uma melhor performance. Para estes autores, um ambiente com menor incerteza sugere estratégias defensivas, baseadas na busca por eficiência e implementadas a partir de estruturas mais centralizadas e padronizadas.

Por outro lado, um maior grau de incerteza sugere estratégias prospectivas, orientadas à eficácia e customização dos serviços, o que exige estruturas mais descentralizadas e processos menos padronizados. No entanto, embora defendam a importância do ajuste entre os fatores que compõem o modelo, os autores não conseguiram identificar de forma conclusiva a relação entre tal ajuste e a performance portuária, sugerindo que a mesma é influenciada por vários outros fatores, sejam internos e/ou externos ao porto. Pode-se inferir que, entre tais fatores, esteja a dependência da trajetória, a qual é explorada no presente estudo (BALTAZAR; BROOKS, 2006).

\section{Procedimentos metodológicos}

A escolha da metodologia a ser adotada em uma pesquisa pode ser qualitativa e/ou quantitativa e qualquer projeto pode ser abordado a partir de uma dessas abordagens. Ainda, de acordo com a referida autora, não há uma metodologia mais apropriada para cada tipo de projeto. No entanto, esta deve ser coerente com a formulação do problema, com os objetivos e com as limitações da pesquisa (REMLER; VAN RYZIN, 2011; SCOTT; GARNER, 2013).

Dadas suas particularidades, o presente estudo se caracteriza como uma pesquisa qualitativa. Para Hair Jr., Bush e Ortinau (2000), a pesquisa qualitativa se concentra em dados provenientes de amostras pequenas e deve ser aplicada nos seguintes casos: no processo de identificação de 
problemas e de oportunidades de negócios; na obtenção de fatores motivacionais, emocionais, atitudinais e pessoais, que influenciam o ambiente dos negócios; no processo de construção de teorias e de modelos; para desenvolver instrumentos de mensuração confiáveis e válidos; para determinar a eficácia das estratégias de marketing; e para analisar cenários de mercado para a introdução de novos produtos e/ou serviços.

Sendo assim, para operacionalizar a pesquisa, foi implementado um estudo de caso pois, segundo Yin (2009), trata-se de uma investigação empírica de um fenômeno contemporâneo dentro de seu contexto real, especialmente indicado quando os limites entre o fenômeno e o contexto não estão claramente definidos e essa é a situação evidenciada no presente estudo. Além disso, o método escolhido torna-se pertinente por ser muito difícil separar o modelo de governança e seus reflexos sobre a cadeia logístico-portuária, devido ao seu dinamismo e à possibilidade de influência mútua. Em outras palavras, por vezes torna-se difícil separar as causas e consequências da governança portuária e isolar o papel da dependência da trajetória nesse contexto. Esta dificuldade justifica a adoção de uma postura interpretativista, o uso de uma abordagem qualitativa e o desenvolvimento de um estudo de caso, considerando a governança portuária exercida no Porto de Valência (Espanha) a partir da Teoria da Path Dependence.

Dentre os tipos de estudos de caso classificados por Yin (2009), o que mais se encaixa neste trabalho é o estudo de caso único, de caráter explanatório. Pretendeu-se, portanto, que o caso estudado possa ser traduzido em explanações utilizáveis em outras situações (análises acerca da governança portuária em portos com outras especificidades).

Para a realização do estudo de caso, empregou-se a pesquisa documental (SCOTT; GARNER, 2013), uma vez que foram utilizados dados secundários, obtidos junto ao Porto de Valência, além de observação direta (HENNINK; HUTTER; BAILEY, 2011) de um dos pesquisadores no referido Porto. Como mencionado anteriormente, os dados foram analisados qualitativamente e de modo interpretativo, por meio da análise de conteúdo (BARDIN, 2004; SCHREIER, 2012), tendo-se como base os pressupostos da Teoria da Path Dependence.

\section{A governança portuária no porto de valência a partir da teoria da Path Dependence}

A Autoridade Portuária de Valência (APV) é um órgão público responsável pela gestão de três portos estatais localizados ao longo de 80 quilômetros na costa mediterrânea oriental espanhola: Valência, Sagunto e Gandia. Tais Portos são explorados mediante uma marca comercial registrada, denominada Valenciaport.

O crescimento constante da movimentação de contêineres no Porto de Valência nos últimos anos resultou em um aumento no volume de serviços relacionados com os mesmos, sobrecarregando os procedimentos e práticas executados no porto. Isso, associado à crescente 
necessidade de cumprir os requisitos internacionais de segurança e proteção (ISPS Code), tem incentivado o desenvolvimento de novas soluções para a coordenação dos atores da comunidade portuária e para a gestão da qualidade das operações envolvendo o porto. Entre essas soluções, destacam-se, na área de Tecnologia da Informação, o desenvolvimento do Port Community System (PCS ou Sistema de Comunidade Portuária) do Porto de Valência e, na área da qualidade, o projeto da Marca de Garantia do Porto. Esses dois projetos começaram a ser desenvolvidos no início dos anos 90 e são pilares fundamentais da governança do referido Porto.

O PCS de Valência (valenciaportpcs.net) é uma plataforma na qual toda a comunidade portuária compartilha informações, permitindo o controle aduaneiro de contêineres de modo automático e sem papéis; a entrada e liberação dos contêineres por meio de portões (gates) automáticos; a notificação online das operações; o maior controle e rastreamento das cargas e a disponibilização de informações como apoio à gestão, entre outros aspectos (LLOP, 2007). Já a Marca de Garantia (LÓPEZ; POOL, 1998), trata-se de um sistema de qualidade para a comunidade portuária a partir do qual as empresas (usuários da marca de garantia) aceitam adequar seus procedimentos aos requisitos estabelecidos no regulamento de uso com vistas a se diferenciarem de seus competidores, darem maior qualidade de serviço aos seus clientes e se beneficiarem de ações comerciais de promoção da marca.

Tanto o PCS quanto a Marca de Garantia vem sendo aprimorados ao longo dos anos com a adequação de procedimentos e regulamentação, e com a inclusão de novos atores, tudo isso permitindo o alinhamento da cadeia logístico-portuária e gerando uma melhoria nas operações do porto (aumento da eficiência, redução de custos etc.). Mas, por outro lado, a forma de comunicação e o modo de operar definido pela governança portuária, a partir do PCS e da Marca de Garantia, induzem os atores a procederem de forma mimética, tornando-os parecidos entre si, dependentes entre si e condicionados pelos instrumentos da governança.

Nesse sentido, Arthur (1989; 1990; 1994; 1996) e Liebowitz e Margolis (1995) mencionam quatro consequências resultantes do processo Path Dependence que, embora relacionadas, são diferentes entre si: i) o aumento de retornos; ii) o auto-reforço; iii) o feedback positivo; e iv) o 'trancamento' (lock-in). O aumento de retornos, para Arthur (1989, 1994; 1996) e Liebowitz e Margolis (1995), faz com que quanto mais se aprofunde determinada escolha, neste caso o PCS e a Marca de Garantia, mais aumentem seus benefícios, gerando um processo virtuoso (LICHA, 2004). Já o auto-reforço diz respeito ao conjunto de forças complementares que dão sustentação à escolha. Isso se percebe nas ações da cadeia logístico-portuária (capacitação dos atores, melhoria de serviços específicos etc.). O feedback positivo está relacionado ao efeito que o benefício gera a um terceiro (externalidade) sem que o mesmo incorra em custos. É o caso do menor tempo para a liberação de contêineres de importação, que pode beneficiar tanto importadores como prestadores de serviços 
logísticos, mesmo aqueles que não fazem parte das iniciativas citadas (PCS e Marca de Garantia) e não suportam os investimentos decorrentes das mesmas. Finalmente, o lock-in, que, de acordo com Licha (2004), significa que, quando uma estrutura é alcançada, não se consegue sair do processo de forma endógena, sem intervenção externa - em outras palavras, o processo está 'trancado' ('aprisionado'). No caso do Porto de Valência, seria muito difícil romper com o processo de aprofundamento das iniciativas em curso e isso não aconteceria de forma espontânea.

No que diz respeito ao grau de dependência (LIEBOWITZ; MARGOLIS, 1995), a Marca de Garantia do Porto de Valência pode ser classificada como de segundo grau, uma vez que a decisão de arrolar todos os parceiros ao redor da marca em termos da qualidade implicou na necessidade de estruturar um sistema que pudesse manter a governança da Marca. Foi necessária a criação de uma forma de auditoria da qualidade e verificação do andamento das ações dos parceiros, o que demanda recursos da governança do porto, podendo afetar as condições competitivas.

No momento da criação da marca não havia o conhecimento exato de quanto isso demandaria recursos no futuro. Assim, em uma análise contra factual, é de se pensar que as estruturas das auditorias terceirizadas e do próprio sistema internacional ISO poderiam trazer resultados similares, garantindo os sistemas da qualidade dos membros sem, contudo, retirar recursos do porto. Porém, atualmente é muito difícil ou custoso abandonar essa trajetória, considerando-se que muitos sistemas e procedimentos foram definidos de acordo com as demandas da Marca de Garantia. Estruturas de auxílio se formaram e algumas externalidades foram criadas, motivando a atuação dos atores no sentido de melhorar o modelo escolhido.

A mesma lógica pode ser vista no PCS, pois a utilização de documentos eletrônicos padronizados e a uniformização de procedimentos, tudo isso condicionado a uma dependência das peculiaridades dos sistemas informacionais, induzem todos os membros da cadeia a trabalharem de forma semelhante, melhorando sistematicamente, mas seguindo um modelo previamente definido e fomentado pela Autoridade Portuária. Portanto, somente será mudada a forma de trabalho ou mesmo criada uma inovadora se a governança assim entender. E este fato é improvável, devido aos custos e esforços investidos, às complementaridades existentes e aos reforços positivos. Dado esse contexto, as perguntas que se impõem são as seguintes: As iniciativas citadas (PCS e Marca de Garantia), considerando os benefícios que geram e os custos de sua manutenção, favorecem a competitividade do Porto de Valência? O Porto pode perder a competitividade ao permanecer 'trancado' ou 'aprisionado' no curso definido por essas iniciativas?

Analisando o PCS e a Marca de Garantia a partir do modelo de governança (Matching Framework) proposto por Baltazar e Brooks (2006), e considerando um possível aprofundamento dessas iniciativas, decorrente da lógica da Path Dependence, pode-se dizer que tanto a Marca de Garantia quanto o PCS tratam-se da busca por eficiência dentro de um modelo preestabelecido. Isso 
pode ser associado a uma estratégia defensiva (MILES; SNOW, 1978 apud BALTAZAR; BROOKS, 2006), a qual funciona melhor em ambientes com menor grau de incerteza e pode ser implementada mediante uma estrutura mecanicista. Por outro lado, esse tipo de trajetória pode dificultar a implementação de estratégias prospectivas e de customização dos serviços, as quais necessitariam de estruturas mais flexíveis (orgânicas).

Esse 'trancamento' (ou 'aprisionamento') no curso estabelecido (estratégia e estrutura) irá mantendo os benefícios até o limite de produtividade, mas o fato é que, se portos concorrentes procederem de forma inovadora e melhor nesses atributos (PCS e Marca), que atualmente diferenciam o Porto de Valência, tornar-se-ão competidores mais eficazes enquanto Valência não conseguir retornar a um melhor curso de eficiência e lucro.

Portanto, as inovações realizadas que atualmente diferenciam e concedem ao porto de Valência o caráter de inovador e a condição de benchmarking internacional poderão prendê-lo em um rumo engessado que pode retirar suas melhores condições competitivas no futuro. É o risco do primeiro a se mover, que pode ter desvantagem no futuro por cultivar em excesso as coisas que deram certo no passado sem capitalizar seus benefícios (MUELLER, 1997).

Para evitar o 'trancamento' da estrutura e da estratégia em um curso desfavorável ao desempenho portuário, sugere-se ao porto uma reavaliação constante dos elementos do modelo de governança proposto por Baltazar e Brooks (2006), especialmente o monitoramento das condições ambientais (Figura 2).

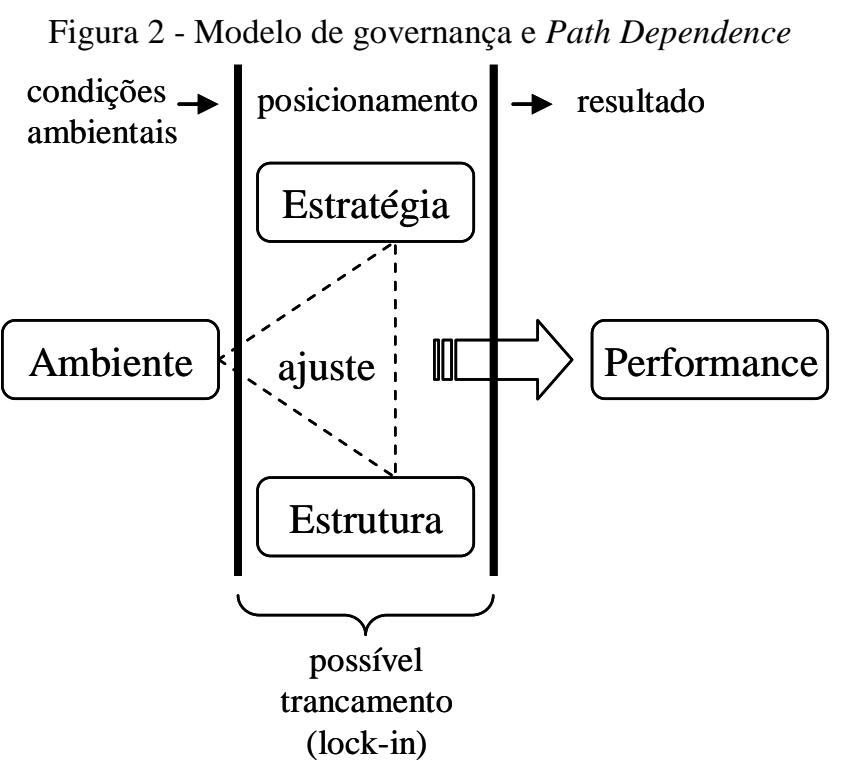

Fonte: Elaborada pelos autores a partir do modelo de Baltazar e Brooks (2006)

O objetivo principal, dadas as condições verificadas no ambiente (com menor ou maior grau de incerteza), é reavaliar constantemente a estratégia estabelecida (reativa ou prospectiva) e a estrutura de governança (mecanicista ou orgânica), criando um ajuste que favoreça a performance e a competitividade portuária, evitando-se o 'trancamento' ou o 'aprisionamento' resultante de 
decisões e práticas implementadas no passado.

A Figura 3 apresenta as diferentes combinações possíveis entre os elementos que compõem cada componente (ambiente, estratégia e estrutura) do modelo proposto por Baltazar e Brooks (2006).

Figura 3 - Possíveis combinações entre os elementos de cada componente do modelo de governança

\begin{tabular}{|c|c|c|c|c|}
\hline Ambiente & Estrutura & Estratégia & Ajuste & Desempenho \\
\hline+ incerteza & Orgânica & Prospectiva & Sim & Melhor \\
\hline+ incerteza & Mecanicista & Prospectiva & Não & Pior \\
\hline+ incerteza & Orgânica & Reativa & Não & Pior \\
\hline+ incerteza & Mecanicista & Reativa & Não & Pior \\
\hline - incerteza & Orgânica & Prospectiva & Não & Pior \\
\hline - incerteza & Mecanicista & Prospectiva & Não & Pior \\
\hline - incerteza & Orgânica & Reativa & Não & Melhor \\
\hline - incerteza & Mecanicista & Reativa & Sim & Melhor \\
\hline
\end{tabular}

Fonte: Elaborada pelos autores a partir do modelo de Baltazar e Brooks (2006)

Analisando-se a Figura 3, percebe-se que um modelo ajustado (e que favorece a performance portuária) ocorre quando há a combinação entre um ambiente com maior incerteza, uma estrutura orgânica e uma estratégia prospectiva, ou um elemento de menor incerteza, uma estrutura mecanicista e uma estratégia defensiva.

Uma possível mudança nas condições ambientais, associada a um possível 'trancamento' em determinados tipos de estratégia e estrutura, pode levar a um desajuste, prejudicando a performance portuária, conforme apresentado na Figura 4.

Figura 4 - Possíveis combinações entre os elementos de cada componente do modelo de governança

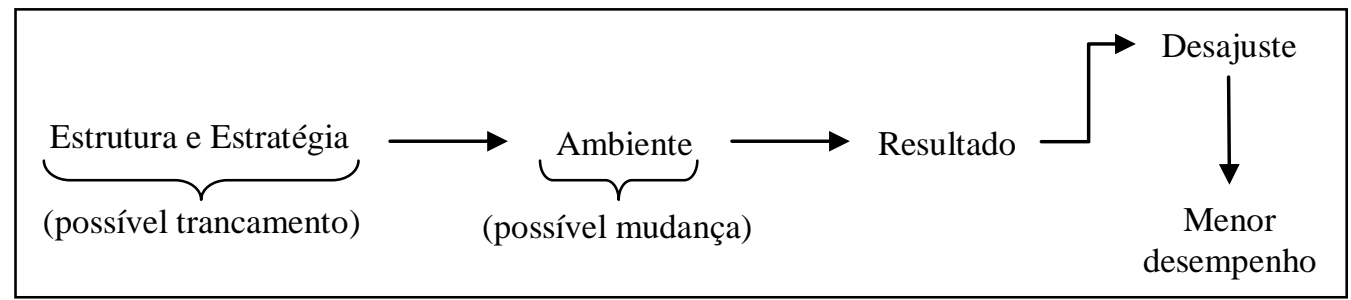

Fonte: Elaborada pelos autores a partir do modelo de Baltazar e Brooks (2006)

Portanto, é necessária uma constante avaliação das condições ambientais, bem como da estrutura e da estratégia existentes, visando um maior ajuste que favoreça o desempenho portuário.

\section{Considerações finais}

A Teoria da Path Dependence revela-se útil para a análise de uma série de situações empresariais, nas quais os eventos históricos (passados) apresentam importância no desenvolvimento e sucesso atual e futuro das organizações. No presente estudo, especificamente, foi explorada a relação entre a governança portuária e a Path Dependence.

Com o estudo de caso realizado junto ao Porto de Valência (Espanha), referência mundial em governança portuária, identificou-se que as ações de governança que hoje diferenciam o Porto e 
apóiam sua competitividade (MILAN; VIEIRA, 2011), podem, no futuro, deixar o Porto 'trancado' (ou 'aprisionado') em um curso desfavorável, ou seja, em uma condição de desajuste entre as estratégias estabelecidas e a estrutura definida para o alcance destas estratégias ou, principalmente, entre estes dois fatores (estratégia e estrutura) e o ambiente competitivo em que o Porto está inserido.

Todavia, Brooks e Cullinane (2006) salientam que ainda não há evidências conclusivas sobre a relação entre modelos de governança e performance portuária, uma vez que o tema ainda não foi suficientemente estudado. Aliás, tais temas merecem atenção dos pesquisadores. De qualquer modo, para os autores, quando são implementados modelos de governança desajustados, não se pode esperar uma performance portuária em nível ótimo.

Portanto, uma vez que esse desajuste pode ser causado pelo 'trancamento' (ou 'aprisionamento') de um porto em um determinado curso estratégico, e o mesmo pode ser explicado pela Path Dependence, esta teoria mostra-se útil para a análise da governança portuária, no sentido de verificar se o porto encontra-se em uma trajetória adequada, tangibilizada pelas suas decisões estratégicas e práticas ou ações implementadas, para sua competitividade presente e futura em relação às forças (internas e externas ao porto) e, principalmente, às mudanças do macroambiente que o cerca.

\begin{abstract}
The ports have been increasingly studied as enterprise clusters and an aspect that emerges from the literature is the coordination of actors belonging to the clusters, the port governance. Most of studies seeking to relate the port governance model to the performance ports, but the results are not conclusive since the performance port also depends on a number of other factors. Among such factors are past events, the previous choices made by the port, that impact their present and futures competitiveness. Therefore, port governance can be studied from the perspective of the path dependence. Thus, this study aimed to analyze the innovations related to port governance in the Valencia Port (Spain) from the assumptions of Path Dependence Theory. The evidences indicate that the Port has achieved positive results as a consequence of their governance actions. However, it is suggested a constants environmental monitoring to avoid the possible locking of the Port in an unfavorable trend.
\end{abstract}

Key-words: port governance; path dependence theory; port of valencia.

\title{
Referências
}

AKABANE, G.K.; GONÇALVES, M.A.; SILVA, T.R. A importância do modelo de autoridade portuária como opção no planejamento logístico: uma pesquisa exploratória. In: VIEIRA, G.B.B.; SANTOS, C.H.S. (org.). Logística e gestão portuária: uma visão ibero-americana. Caxias do Sul: Educs, 2008. cap. 3, p.49-61.

ARTHUR, W.B. Competing technologies, increasing returns, and lock-in by historical events. Economic Journal, v.99, n.394, p.116-131. 1989.

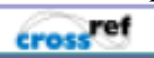

ARTHUR, W.B. Positive feedbacks in the economy. Scientific American, v.262, p.92-99, 1990. 
ARTHUR, W.B. Increasing returns and path dependence in the economy. Ann Harbor: University of Michigan Press, 1994.

ARTHUR, W.B. Increasing returns and the two worlds of business. Harvard Business Review, Jul-Ago 1996.

BALTAZAR, R.; BROOKS, M.R. Port governance, devolution and the Matching Framework: a configuration theory approach. Research in Transportation Economics, v.17, n.1, p.379-403, 2006.

\section{cross ref}

BARDIN, L. Análise de conteúdo. Lisboa: Edições 70, 2004.

BROOKS, M.R. The governance structure of ports. Review of Network Economics, v.3, n.2, p.168-183, 2004.

\section{cross $r$}

BROOKS, M.R.; CULLINANE, K. Conclusions and research agenda: devolution, port governance and port performance. Research in Transportation Economics, v.17, n.1, p. 631-660, 2006.

\section{cross ${ }^{\text {ref }}$}

DAVID, P.A. Path dependence, its critics and the quest for historical economics. In: GARROUSTE, P.; IOANNIDES, S. (eds.). Evolution and path dependence in economic ideas: past and present. Cheltenham: Edward Elgar Publishing, 2000 .

DE LANGEN, P.W. Governance in seaport clusters. Journal of Maritime Economics and Logistics, v.6, n.4, p.141$156,2004$.

\section{cross ref}

DE LANGEN, P.W.; NIJDAM, M.N; van der HORST, M.R. Port performance indicators in ports. Journal of Maritime Research, v.4, n.1, p.23-36, 2007.

DOSI, G.; NELSON, R.R. An introduction to evolutionary theories in economics. Evolutionary Economics, v.4, n.3, p.153-172, 1994.

\section{cross ref}

ESPO - European Sea Ports Organisation. Annual report 2007-2008. Brussels: ESPO, 2008. Disponível em: www.espo.be/downloads/archive/8bf1fded-66f6-4e0f-8204-7e52754b9b69.pdf.Acesso em: 13 abr. 2012.

FRANKEL, E.G. Strategic planning applied to shipping and ports. Policy and Management, v.16, n.2 p.123-132, 1989.

cross $r$

GEIGER, A. Modelo de governança para apoiar a inserção competitiva de arranjos produtivos locais em cadeias globais de valor. Tese de Doutorado. Programa de Pós-Graduação em Engenharia de Produção (PPGEP). Universidade Federal do Rio Grande do Sul (UFRGS). Porto Alegre, 2010.

HAIR Jr., J.F.; BUSH, R.P.; ORTINAU, D.J. Marketing research: a practical approach for the new millennium. Boston: Irwin/McGraw-Hill, 2000.

HEAVER, T.D.; MEERSMAN, H.; MOGLIA, F.; van de VOORDE, E. Do mergers and alliances influence European shipping and port competition? Maritime Policy and Management, n. 27, p. 363-373, 2000.

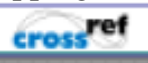

HENNINK, M.; HUTTER, I.; BAILEY, A. Qualitative research methods. Thousand Oaks: Sage Publications, 2011.

LICHA, A.L. Dependência da trajetória, irreversibilidade e o papel da história na seleção de tecnologias. Revista de Economia, v.30, n.1, p.107-127, 2004. 
LIEBOWITZ, S.J.; MARGOLIS, S. Path dependence, lock-in, and history. Journal of Law, Economics \& Organization, v.11, n.1, p.205-226, 1995.

LLOP, M. Actions towards enhancing the control of operations in ports. In: Proceedings of the Workshop on Multimodal Transport and ICT: Results and Recommendations. Quaderni della Direzione Generale Reti Infrastrutturali, Logistica e Sistemi di Mobilità, n.2, p.87-98, 2007.

LÓPEZ, C.R.; POOLE, N. Quality assurance in the maritime port logistics chain: the case of Valencia, Spain. Supply Chain Management, v.3, n.1, p.33-44, 1998.

cross ref

MARTIN, J.; THOMAS, B.J. The container terminal community. Maritime Policy and Management, v.28, n.3, p.279-292, 2001.

cross ref

MEERSMAN, H; MOGLIA, F; van de VOORDE, E. Mergers and alliances in liner shipping: what do European port authorities have to fear? In: Proceedings of the 1999 Halifax Conference. Liner Shipping: What's next? p.204-220, 1999.

MILAN, G.S.; VIEIRA, G.B.B. Proposição de um modelo conceitual em torno da prática da governança em cadeias logístico-portuárias. Revista Gestão Industrial, v. 7, n. 4, p. 154-174, 2011.

cross ref

MUELLER, D. First-mover advantages and path dependence. International Journal of Industrial Organization, v.15, n.6, p.827-50, 1997.

cross ref

NELSON, R.; WINTER, S. Introduction. In: An Evolutionary Theory of Economic Change. Boston: Harvard Press, 1982.

NOTTEBOOM, T. Inland waterway transport of containerised cargo: from infancy to a fully-fledged transport mode. Journal of Maritime Research, v.4, n.2, p.63-80, 2007.

NOTTEBOOM, T; WINKELMANS, W. Reassessing public sector involvement in European seaports. International Journal of Maritime Economics, v.2, n.3, p.242-259, 2001.

cross ref

OECD - Organisation for Economic Co-Operation and Development. Principles of Corporate Governance. Paris: OECD Publications Service, 2004. Disponível em: www.oecd.org/dataoecd/32/18/31557724.pdf. Acesso em: 13 abr. 2012.

REMLER, D.K.; VAN RYZIN, G.G. Research methods in practice: strategies for description and causation. Thousand Oaks: Sage Publications, 2011.

ROBINSON, S.E.; MEIER, K.J. Path dependence and organizational behavior: bureaucracy and social promotion. The American Review of Public Administration, 2006. Disponível em: www. sagepublications.com. Acesso em: 14 abr. 2007.

SCHREIER, M. Qualitative content analysis in practice. London: Sage Publications, 2012.

SCOTT, G.; GARNER, R. Doing qualitative research: designs, methods, and techniques. $1^{\text {st }}$ edition. Upper Saddle River: Pearson, 2013.

SUYKENS, F; van de VOORDE, E. A quarter of a century of port management in Europe: objectives and tools. Maritime Policy and Management, v.25, n.3, p.251-261, 1998.

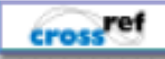

UNCTAD - United Nations Conference on Trade and Development. Strategic Planning for Port Authorities. United Nations: Geneva, 1993. 
van de VOORDE, E.; WINKELMANS, W. A general introduction to port competition and management. In: Port competitiveness: an economic and legal analysis of the factors determining the competitiveness of seaports.

Antwerpen, De Boeck, p.1-16, 2002.

VEBLEN, T. Imperial Germany and the revolution. New Jersey: New Brunswick, 1990.

VIEIRA, G.B.B. MILAN, G.S.; BASSANESI, M.M.R.; PASA, G.S. Governança em clusters portuários: proposição de um modelo conceitual. In: XVII Simpósio de Engenharia de Produção - SIMPEP. Anais... Bauru-SP, 2010.

WILK, E.O. O uso de uma abordagem de "core competences" na formulação de um "portfólio" de diversificação. Dissertação de Mestrado. Programa de Pós-Graduação em Administração (PPGA). Universidade Federal do Rio Grande do Sul (UFRGS). Porto Alegre, 1997.

WINKELMANS, W. One day conference on current trends and practices in the organisation, operation and management of ports and port terminals. Institute of Transport and Maritime Management Antwerp. University of Antwerp. Thessaloniki, Greece, 2008. Disponível em: WWW.hermes.civil.auth.gr/pgtransport/docs/ Winkelmans_PORT_GOVERNANCE_and_SRM_2008.pdf. Acesso em: 12 abr. 2012.

YIN, R.K. Case study research: design and method. $4^{\text {th }}$ edition. New York: Sage Publications, 2009.

\section{Dados dos autores:}

Nome completo: Guilherme Bergmann Borges Vieira

Filiação institucional: Universidade de Caxias do Sul

Departamento: Centro de Ciências Econômicas, Contábeis e de Comércio Internacional

Função ou cargo ocupado: professor e pesquisador

Endereço completo para correspondência: Rua Francisco Getúlio Vargas, 1130 - Caxias do Sul - RS

- 95070-560

Telefones para contato: (54) 3218-2100

e-mail: gbbvieir@ucs.br

Nome completo: Roberto Birch Gonçalves

Filiação institucional: Universidade de Caxias do Sul

Departamento: Centro de Ciências Econômicas, Contábeis e de Comércio Internacional

Função ou cargo ocupado: professor e pesquisador

Endereço completo para correspondência: Rua Francisco Getúlio Vargas, 1130 - Caxias do Sul - RS

- 95070-560

Telefones para contato: (54) 3218-2100

e-mail:rbgoncal@ucs.br

Nome completo: Gabriel Sperandio Milan

Filiação institucional: Universidade de Caxias do Sul

Departamento: Programa de Pós-Graduação em Administração 
Função ou cargo ocupado: professor e pesquisador

Endereço completo para correspondência: Rua Francisco Getúlio Vargas, 1130 - Caxias do Sul - RS

- 95070-560

Telefones para contato: (54) 3218-2100

e-mail: gabmilan@terra.com.br

Nome completo: Francisco José Kliemann Neto

Filiação institucional: Universidade Federal do Rio Grande do Sul

Departamento: Programa de Pós-Graduação em Engenharia de Produção

Função ou cargo ocupado: professor e pesquisador

Endereço completo para correspondência: Av. Osvaldo aranha, 99, 5ªndar - Bairro Bom Fim -

Porto Alegre - RS - CEP 90035-190

Telefones para contato: (51) 33083491

e-mail:kliemann@producao.ufrgs.br

Submetido em: 22/11/2012

Aceito em: 01/10/2013 